\title{
Deficits in mouse-killing following trigeminal lesions in the rat
}

\author{
STEPHEN L. WELLE and GARY D. COOVER \\ Northern Illinois University, DeKalb, Illinois 60115
}

\begin{abstract}
Lesions of the trigeminal somatosensory system were produced in adult male hooded rats that would spontaneously kill C57B1/6 mice (i.e., natural killers). The mouse-killing behavior was assessed by the speed of the initial attack (1/latency in seconds), reflecting the release of the bchavior, and the speed and efficiency (1/number of attacks to kill) of killing. Tenminute test sessions were given once every 2-4 days, 3-4 times prior to trigeminal or control surgery and again 5-6 times following surgery. In three experiments, it was found that lesions of the medial ventrobasal thalamic complex (VBm), trigeminal sensory root, or infraorbital branch of the trigeminal nerve decreased the speed and efficiency of killing. In rats that were induced to kill by food deprivation and were tested while food-deprived, the trigeminal sensory root lesions had the same effects, although the impairment of killing speed was not as marked. Only the VBm lesions decreased the initial attack speeds, and this deficit was attenuated by 2 weeks following surgery. It is concluded that perioral somatosensory cues normally guide the mouse-killing behavior but are not necessary for initiation of attack behavior.
\end{abstract}

Mouse-killing, which consists of a stereotyped biting attack directed at the cervical region, occurs spontaneously in some laboratory rats. Rats that do not spontaneously kill may be induced to attack mice by food deprivation (Paul, Miley, \& Baenninger, 1971) or by lesions of the septum (Miczek \& Grossman, 1972) or the olfactory tract (Karli, Vergnes, \& Didiergeorges, 1969). Mouse-killing decreases only very slowly when the opportunity to eat the dead mouse is denied the rat (Kulkarni, 1968; Myer \& White, 1965; Van Hemel \& Myer, 1970). The behavior has also appeared to be highly resistant to most forms of sensory deprivation (Alberts \& Friedman, 1972; Karli et al., 1969). However, it was recently reported that transection of the infraorbital branch of the trigeminal nerve, which subserves perioral facial somatosensation, abolished attack behavior in some rats and decreased the efficiency of killing in others (Gregoire \& Smith, 1975). It has also been reported that lidocaine anesthesia of the mystacial vibrissae can increase the latencies of attacking and killing (Thor \& Ghiselli, 1975).

In addition to its role in mouse-killing, the trigeminal system is important in ingestive behaviors. Zeigler and Karten (1974) found that lesions along

This study was a partial fulfillment of the first author's Master's requirement. Portions of the data were presented at the meeting of the Midwestern Psychological Association, Chicago, May 1977. We gratefully acknowledge the scholarly assistance of Raymond Ditrichs, Carlton Lints, and James Willott. Requests for reprints should be sent to Gary Coover, Department of Psychology, Northern Illinois University, DeKalb, Illinois 60115. the trigeminal lemniscus (LTr) or in the medial twothirds of the ventrobasal thalamic complex (VBm) in which LTr fibers terminate can cause aphagia, adipsia, finickiness, and excessive food spillage. These deficits are similar to those induced by lateral hypothalamic and nigrostriatal lesions (Marshall, Richardson, \& Teitelbaum, 1974). Since mouse-killing behavior is temporarily abolished by lateral hypothalamic lesions (Karli et al., 1969), this behavior may provide another tool for elucidation of the mechanisms disrupted by trigeminal, lateral hypothalamic, and nigrostriatal lesions.

The present study examines the effects of peripheral and central ( $\mathrm{VBm})$ lesions of the trigeminal system on mouse-killing by spontaneous, or natural, killers. The previous studies relating trigeminal denervation to deficits in killing (Gregoire \& Smith, 1975; Thor \& Ghiselli, 1975) involved the use of rats that had to be food-deprived before they would kill. We examined the speeds of initial attack and of killing and the number of attacks to kill, and the recovery of performance on these measures following surgery. We found that trigeminal lesions disrupted the efficiency of killing whether the lesions were of the infraorbital branch, the trigeminal sensory root, or the ventrobasal thalamic complex (VBm). However, only the VBm lesions (Experiment 1) decreased the speed of initial attack, and this was a temporary effect.

\section{GENERAL METHODS}

\section{Subjects}

The original subject pool consisted of 142 male hooded rats 
bred in the animal colony of the Psychology Department of Northern Illinois University. At 74-100 days of age, the rats were individually housed in suspended metal cages $(18 \times 20 \times 24 \mathrm{~cm})$ with wire-mesh fronts and floors. Food (Wayne Lab Blox) and water were provided ad lib except when noted below. A 12-h light-dark cycle was used, with lights on at 7 a.m. Room temperature was maintained at $20^{\circ}-23^{\circ} \mathrm{C}$.

\section{Screening}

The subjects were screened for spontaneous killing after at least 2 days of habituation to their new surroundings. An adult C57B1/6 mouse was placed in each cage and left overnight. Rats that killed the mouse during this period were designated natural killers and were maintained on ad-lib food and water for the remainder of the experiment. Of the 142 rats screened, $94(66 \%)$ were natural killers. Data were collected on 57 of these. Of the 37 natural killers for which data were not collected, 7 exhibited inconsistent killing on the trials prior to surgery, 8 died during surgery, 1 was discarded following surgery due to motor deficits (Experiment 3), and 21 were randomly discarded as excess animals.

Food-deprived killers were obtained for Experiment 3 by placing 32 of the nonkillers on a $221 / 2-h$ food-deprivation schedule for 1 week, and then rescreening them $24 \mathrm{~h}$ after the last feeding. This yielded 21 food-deprived killers, 3 of which died during surgery.

\section{Behavioral Testing}

The rats were given three or four mouse-killing tests at 2-4day intervals prior to surgery. Following the trigeminal or control surgery, they were given five or six mouse-killing tests at similar intervals.

Each mouse-killing test consisted of placing the mouse into the cage of the rat for 10 min or until shortly after the kill was completed. The experimenter measured the latency of the initial attack and the latency of the kill to the nearest second with a stopwatch. All instances of attack were counted.

An attack was defined as a contact of the mouse in which the rat attempted to bite in a manner that could clearly injure or kill the mouse. Contacts involving only sniffing, licking, and tugging of the skin were not scored as attacks. Attacks were defined as being separate when the mouse completely disengaged itself or when the rat withdrew completely from the mouse. The time of the kill was defined in Experiments 1 and 2 as the time of cessation of all movement, including breathing, and in Experiment 3 as the time of the audible break of the spinal cord or skull followed immediately by clonic muscle contractions. These measures of time of attack, occurrences of attack, and time of the kill were examined in a comparison of ratings from live and videotape observations; Pearson correlations were above .98 for all three measures.

The possibility of motor damage was examined in all the rats following surgery. A metal probe was inserted into the mouth of each rat after the initial postoperative muricide test and depressed slightly to check for the presence of chewing movements. The ability to gnaw dry food pellets was also used to determine motor involvement in surgical effects.

\section{Surgery and Histology}

Preparation for surgery consisted of an IP injection of $.25 \mathrm{ml}$ atropine sulfate $(.5 \mathrm{mg} / \mathrm{ml})$ prior to anesthetization with Nembutal $(50 \mathrm{mg} / \mathrm{kg})$. The head was fixed for surgery in a Kopf stereotaxic device. The electrodes used in Experiments 1 and 3 were made by coating No. 00 insect pins with Epoxylite, except for $.5 \mathrm{~mm}$ of the tip. Following testing, the brains were fixed in $10 \%$ Formalin. They were blocked, frozen, and sectioned with a Jung Ag freezing microtome. Unstained sections were taken throughout the extent of the lesion and photographed by projecting the image on Kodabromide photographic paper.

\section{Data Analysis}

The data from Experiment 1 were handled in a post hoc descriptive manner because of the small and variable number of subjects showing any killing following thalamic surgery. In Experiments 2 and 3, parametric statistics were applied. Each of the three behavioral scores, for initial attack, for time to kill, and for number of attacks required to kill were analyzed, first for the preoperative trials and then the postoperative trials. Reciprocals of each trial score were used in the analysis: the speed (1/latency in seconds) of initial attack, the speed to kill after the initial attack, and the efficiency ratio (1/number of attacks). Multivariate analyses of variance were first applied to deal with the repeated measures of trials. This approach involved multivariate analyses of successive differences in reciprocal scores on preoperative, or postoperative, trials. With this procedure, a significant main effect of a factor such as Lesions is interpretable as a Lesion by Trials interaction and is described in this manner on the following pages. If the multivariate analysis yielded no such interaction of the explicit factor with trials, then a test was made of any significant change in speed or efficiency over trials. This involved a test of the geometric mean of difference scores against the null hypothesis. Subsequently, the analysis was pursued using univariate analyses of variance of the reciprocal scores averaged over the preoperative, or postoperative, trials.

The postoperative data of Experiments 2 and 3 were also analyzed with covariate analyses, using the score on the last preoperative trial as the predictor. This did not alter the results obtained with the procedures describes above, except in one instance noted in the results of Experiment 3

\section{EXPERIMENT 1}

\section{Procedure}

Twenty-one natural killers were screened on Day 1 and then given three preoperative tests, one each on Days 3,5 , and 8 . On Day 9, the rats were subjected to either bilateral lesions of the VBm of the thalamus $(n=11)$ or a control surgical treatment (shams, $\mathrm{n}=10$ ). Three days later (Day 12), they were given the first of five postoperative tests. Single tests were then given on Days 15, 17, 19, and 22.

Water consumption was recorded daily and body weight every 3rd day. Since some of the VBm rats would not eat enough dry food to maintain strength, $50 \mathrm{~g}$ of a wet food mash (ground Wayne Lab Blox and water) was provided daily to all subjects. Observations were made daily on spontaneous locomotion, food intake, and the amount of food spillage.

The VBm lesions were produced by aiming the electrode according to the following coordinates: $3.0 \mathrm{~mm}$ posterior to bregma, $2.5 \mathrm{~mm}$ bilateral to the midline suture, and $7.4 \mathrm{~mm}$ below the surface of the skull. The head was fixed in the stereotaxic apparatus with the surface of the skull level between lambda and bregma. Anodal lesions were made in the VBm subjects by passing $1.5 \mathrm{~mA}$ of current for $25 \mathrm{sec}$, with a rectal probe as the cathode.

\section{Results}

Lesions of the VBm produced a clear decrease in mouse-killing behavior. The control subjects maintained mouse-killing behavior at the level of performance exhibited on the last trial prior to surgery: initial attack within $10 \mathrm{sec}$ and killing within an additional $45 \mathrm{sec}$ with six or fewer attacks. Only 5 of the 11 rats with VBm lesions succeeded in killing a mouse during the five postoperative trials, which is below the control level, $\chi_{1}^{2}=8.10, \mathrm{p}<.01$.

Figure 1 presents the percentage of rats with lesions 


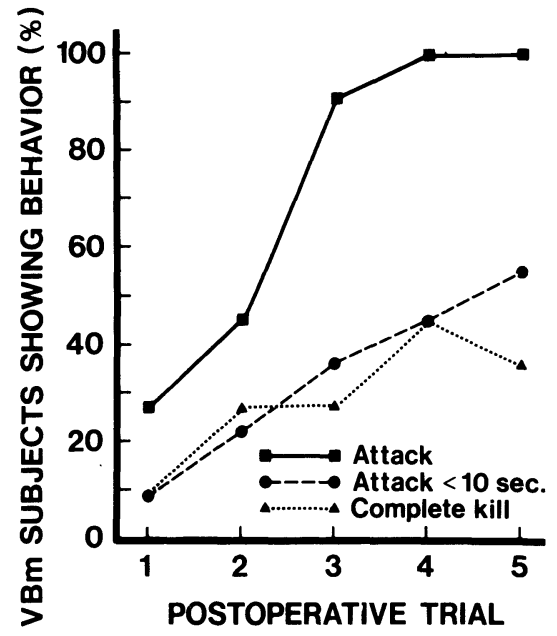

Figure 1. Percentage of natural killers with VBm lesions attacking, attacking in less than $10 \mathrm{sec}$, and completing the kill on postoperative trials. The 10 -min trials were given once every 2-3 days. The control operated rats performed at $100 \%$ on each behavior.

attacking and killing the target mouse on each postoperative trial. Attack behavior returned in all of the $\mathrm{VBm}$ rats, and approached the rapidity of initial attack exhibited by the control subjects (less than 10-sec latency). The speed of initial attack was not significantly lower on the final trial for the lesion group $[\mathrm{M}=.32(\mathrm{SE}=.12)]$ compared to the control group $[\mathrm{M}=.58(\mathrm{SE}=.11)][\mathrm{t}(19)=1.65$, $\mathrm{p}>.05]$, and only 2 of the $11 \mathrm{VBm}$ rats had failed to increase their speed of attack from the fourth to the fifth postoperative trial.

When the rats with VBm lesions attacked the mouse, they always attacked more than once on that trial, and on all subsequent trials. However, even when they killed the mouse, the kill was accomplished with more attacks than required by the control subjects (Table 1). The data in Table 1 are derived from the first three postoperative trials on which an attack was made. While the data for latency to initial attack on these trials demonstrate the recovery of attack behavior, the data for number of attacks to kill provide no evidence of recovery of efficiency of killing over this limited number of trials available for systematic analysis. Comparison of the VBm rats that killed at least once with those that never killed postoperatively does not indicate further differences, according to the latency of attack and efficiency measures in Table 1 . However, four of the five rats that attacked on the first or second postoperative trial also killed at least once postoperatively. These five individuals that attacked on more than three trials postoperatively did not, on the subsequent trials, establish better scores for killing efficiency than they had already established on the first three trials.

The loss of attack in most subjects for the first two trials after VBm lesions was associated with apparent somnolence and akinesis in the home cage prior to the tests. However, presentation of the mouse elicited sniffing and licking of the mouse even though no aggression was displayed. When attack behavior reappeared in the 2 nd week after surgery, the rats would exhibit bouts of attacking throughout the testing period or until they succeeded in killing the mouse. Usually, an attack was terminated by the mouse escaping. Frequently, the rat was able to hold the mouse but not to deliver a killing bite: instead, it would mouthe the mouse, covering it with saliva.

Hypodipsia and weight loss were observed in the VBm group. They consumed only $50 \%$ of their preoperative water intake over the 1 st and 2 nd week following surgery, and lost body weight at the rate of $67 \mathrm{~g}(22 \%$ loss $)$ the 1 st week and $22 \mathrm{~g}$ the 2 nd week. The fact that attacking improved in the 2nd week even though the rats continued to lose weight suggests that the temporary attack deficit was not due to inanition. The control group maintained its

Table 1

Median Initial Attack Latency (in Seconds) and Number of Attacks to Kill for Trials on Which an Attack Occurred

\begin{tabular}{|c|c|c|c|c|c|c|c|c|c|}
\hline & \multirow[b]{3}{*}{$\mathrm{n}$} & \multirow{2}{*}{\multicolumn{2}{|c|}{$\begin{array}{c}\text { Preoperative } \\
\text { Last Trial }\end{array}$}} & \multicolumn{6}{|c|}{ Postoperative } \\
\hline & & & & \multicolumn{2}{|c|}{ First Trial } & \multicolumn{2}{|c|}{ Second Trial } & \multicolumn{2}{|c|}{ Third Trial } \\
\hline & & Median & Range & Median & Range & Median & Range & Median & Range \\
\hline & & \multicolumn{8}{|c|}{ Initial Attack Latency } \\
\hline Control & 10 & 1 & $1-9$ & 2 & $1-5$ & 2 & $1-8$ & 2 & $1-9$ \\
\hline $\mathrm{VBm}$ & 11 & 3 & $1-37$ & 20 & $5-60$ & 10 & $2-60$ & 7 & $1-45^{*}$ \\
\hline VBm Killers & 5 & 7 & $2-37$ & 20 & $5-59$ & 4 & $3-60$ & 5 & $2-45$ \\
\hline \multirow[t]{2}{*}{ VBm No Kills } & 6 & 3 & $1-8$ & 25 & $9-60$ & 17 & $2-45$ & 10 & $1-14 *$ \\
\hline & & \multicolumn{8}{|c|}{ Number of Attacks to Kill } \\
\hline Control & 10 & 1 & $1-4$ & 2 & $1-6$ & 2 & $1-4$ & 1 & $1-6$ \\
\hline VBm & 11 & 1 & $1-4$ & 5 & $4-24$ & 16 & $2-30$ & 27 & $3-68$ \\
\hline VBm Killers & 5 & 2 & $1-4$ & 15 & $4-21$ & 7 & $2-29$ & 28 & $3-68$ \\
\hline VBm No Kills & 6 & 1 & $1-2$ & 15 & $5-24$ & 20 & $12-30$ & 27 & $12-31$ \\
\hline
\end{tabular}

Note-VBm killers are those that killed a mouse on at least one of the five trials following surgery. When VBm rats did not kill, the number of attacks during the 10-min trial were used. *One rat with VBm lesions attacked on only two trials. 
water intake and increased its body weight by $4 \%$ over the 2 weeks.

Histological analyses indicated bilateral lesions of the posterior two-thirds of the ventral thalamic nucleus (VB), with the most consistent and complete damage to the medial three-fourths of this structure (Figure 2). The VBm was thus damaged bilaterally in all subjects. All lesions damaged the greater portion of the posterior thalamic nucleus as well. About half of the lesions encroached on the adjacent portions of one or more of the following structures: the lateral posterior nucleus in the dorsal direction, the medial lemniscus and dorsal portion of the zone inserta in the ventral direction, the anterior portion of the medial geniculate caudally (but damage to the medial geniculate was always unilateral), and the posterior portion of the lateral thalamic nucleus in the rostral direction. The dorsal hippocampus was damaged in all the rats by the electrode track but not by the electrolytic lesions. Nearby structures that were consistently spared included the lateral portion of the medial thalamic nucleus, fasciculus retroflexus, parafascicular nucleus, mammillothalamic tract, Forels fields, internal capsule, and lateral geniculate. The deficits in killing were related to destruction of the $\mathrm{VBm}$ and posterior thalamic nucleus, which together receive all of the input from the trigeminal lemniscus (Smith, 1973). Damage to other structures could not be related to behavioral deficits.

\section{EXPERIMENT 2}

The deficient performance of mouse-killing produced by VBm lesions is similar to that found by Gregoire and Smith (1975) after infraorbital nerve transection and by Thor and Gheselli (1975) after lidocaine anesthesia of the vibrissae. However, we
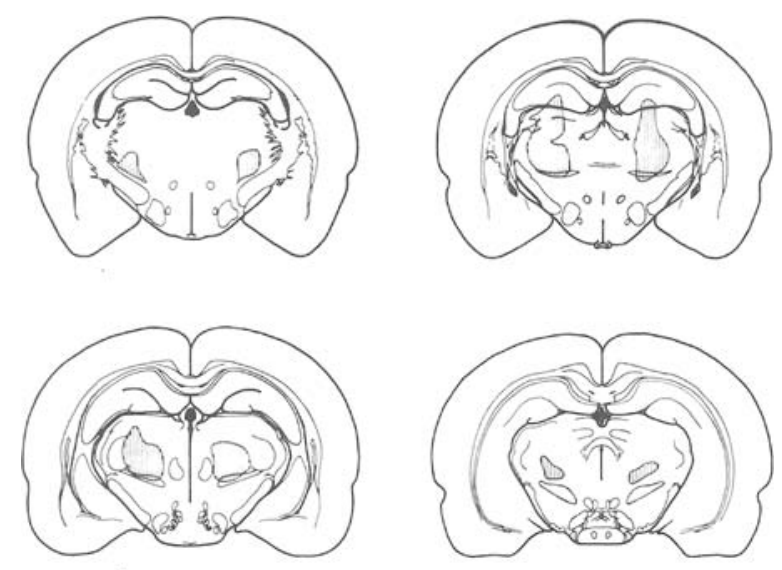

Figure 2. Schematic representation of tissue damage (shaded area) in a rat with a typical VBm lesion. (Adapted from König and Klippel, 1963.)

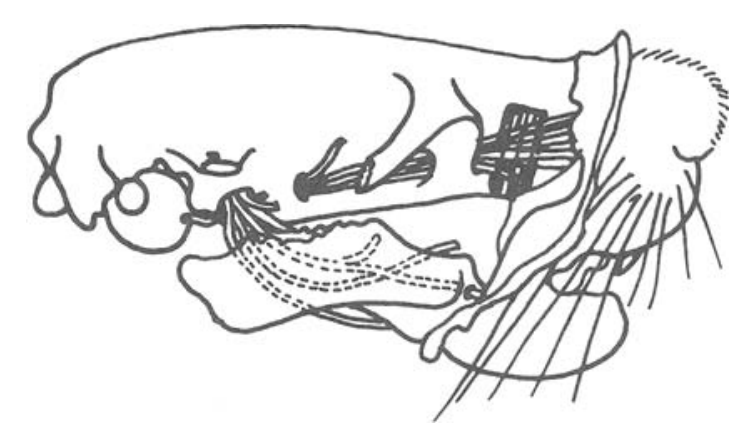

Figure 3. Diagram of a lateral view of the maxillary and mandibular divisions of the trigeminal nerve. Shaded area represents approximate location of interruption of the infraorbital nerve. (Adapted from Greene, 1955.)

found a recovery of attack behavior which Gregoire and Smith did not observe. We thus felt it important to determine the effects of infraorbital transection on our population of natural killers, using our procedure of longer test sessions at longer intertest intervals.

\section{Procedure}

Sixteen natural killers were screened on Day 1 and then given three preoperative mouse-killing tests on Days 3,6 , and 10 . Surgery was performed in the afternoon following the third preoperative test. Six postoperative tests were then given on Days $13,17,20,24,27$, and 31 .

All animals were subjected to shaving of the hair and vibrissae anterior to the eyes, followed by a dorsoventral incision of the skin $5 \mathrm{~mm}$ anterior to each eye. The portion of the maxillary division of the trigeminal nerve that leaves the cranium anterior to the infraorbital fissure was localized (Figure 3). This portion of the maxillary nerve innervates the snout, the upper lip, and the mucous membrane of the mouth (Greene, 1955). This nerve branch was severed bilaterally in the eight rats randomly assigned to the infraorbital transection group, and left intact in the control subjects (shams, $n=8$ ). The wounds were sutured.

\section{Results}

All of the rats completed the kill within every 10-min test session throughout the experiment. Infraorbital transection had no significant effects on the release of mouse-killing behavior, but did impair the performance of killing behavior (Figure 4).

The release of killing, as measured by the speed of initial attack, was not statistically different in the infraorbital and sham groups either prior to or following surgery. However, following surgery, the infraorbital group was significantly slower in completing the kill after the initial attack $[F(1,14)=$ $10.54, \mathrm{p}<.01]$, and the attacks were significantly less effective, as shown by lower mean efficiency ratios across postoperative trials $[\mathrm{F}(1,14)=7.44$, $\mathrm{p}<.05]$. There were no significant trials effects or Groups by Trials interactions for any of the measures over postoperative trials. Thus, there was no evidence of recovery of function in the transected group. 

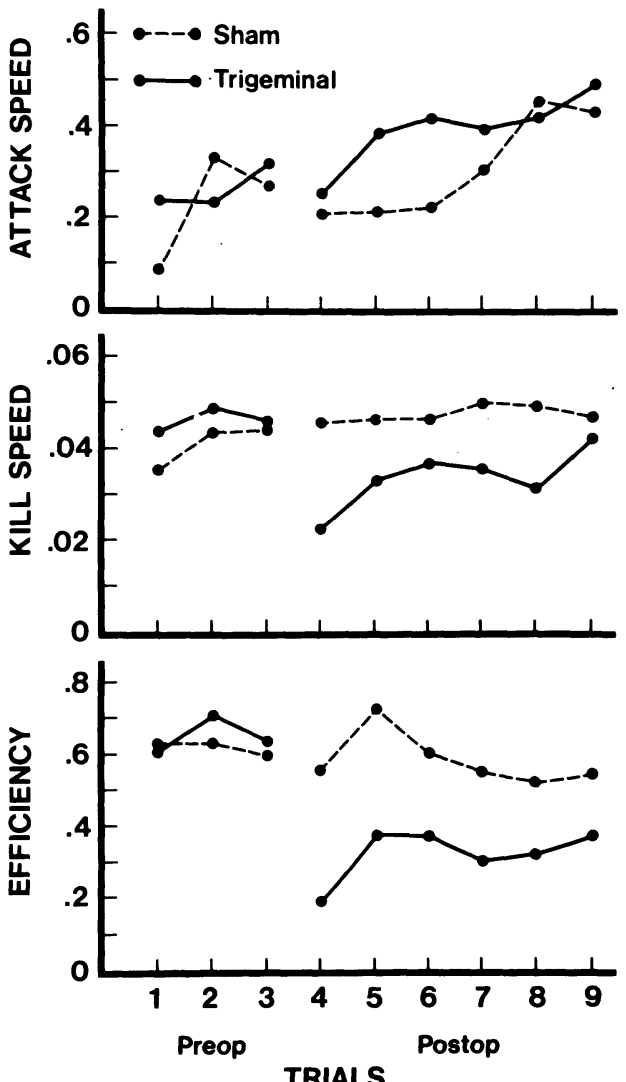

Figure 4. Mean speed (1/latency in seconds) of initial attack, speed to kill after the initial attack, and efficiency (1/number of attacks to complete the kill). Trigeminal damage was made in natural killers by bilateral transection of the infraorbital nerve. Ten-minute trials were given once every 3-4 days, and there was a 3-day recovery period after surgery.

It is concluded that reduced somatic sensitivity of the upper perioral region decreases the efficiency of mouse-killing behavior, but there is no evidence that it interferes with its normal release in natural killers.

Unlike VBm lesions transection of the infraorbital branch did not result in disruption of ingestive behavior. No anorexia or excessive food spillage was observed, and there was no significant loss of weight in the denervated group in comparison with the sham group over the course of the experiment. No gross motor deficits were observed, and the rats were able to chew vigorously and gnaw food pellets on the day following surgery.

Nerve regeneration was not apparent in postmortem examination of the perfused rats.

\section{EXPERIMENT 3}

Transection of the infraorbital nerve decreased the efficiency and speed of killing, but not the initiation of attack behavior. Thus, natural killers, tested with our procedure, were not as severly affected by this peripheral denervation as were Gregoire and Smith's food-deprived killers that were tested following surgery while not food deprived. This discrepancy might not be due only to the difference in subpopulation of rats. For example, Gregoire and Smith used test sessions of only $120 \mathrm{sec}$ duration, a procedure which may not have allowed attacks to occur, thereby depriving the rats of learning experience needed for recovery of function. Thor and Ghiselli (1975) found a mean attack latency of $150 \mathrm{sec}$ after functional denervation by lidocaine anesthesia, albeit their fooddeprived killers were tested while food-deprived and their subpopulation of rats probably included rats that would not have killed when tested under ad-lib conditions. Our natural killers all attacked within $20 \mathrm{sec}$ following infraorbital transections.

The decrease in efficiency and speed of killing produced by infraorbital transections suggest that the same deficits produced by the VBm lesions were due to trigeminal damage. However, the $\mathrm{VBm}$ lesions produced both an additional temporary deficit in initiation of attack, and an apparently more severe deficit in killing. It is not surprising that the VBm lesions would have additional effects, since the VBm lesions damage a pathway involved in taste (Smith, 1973) and produce deficits in acquisition of tactile discriminations (Reyes, Finger, \& Frye, 1973) which we assume are not mediated entirely by facial sensation. However, since the additional effect on initiation of attack was a temporary one, it is not clear to which system(s), trigeminal or other, it should be attributed. It is also not surprising that the VBm lesions would have greater effects on trigeminally mediated functions than are found with infraorbital transections, since the VBm subserves a larger trigeminal sensory field. On the other hand, the VBm lesion does not disrupt all trigeminal input, leaving, for example, the reticular formation input.

In Experiment 3, we lesioned the trigeminal nerve proximal to the convergence of its branches, i.e., at the sensory root near the pons, in order to produce more widespread peripheral denervation without causing extratrigeminal damage. We examined the effects of this lesion on natural killers and also on food-deprived killers tested while food deprived as a precaution against the possibility that the subpopulation of rats would affect the results in a major way. Although total peripheral denervation was not attained, Experiment 3 replicates the finding that subtotal trigeminal denervation reduces the efficiency of, but not the motivation for, attacking. The effects were obtained in both natural and food-deprived killers.

\section{Procedure}

Four groups of rats were employed: natural killers with trigeminal lesions $(n=10)$, natural killers with control operations 
$(n=10)$, deprived killers with trigeminal lesions $(n=10)$, and deprived killers with control operations $(n=8)$. Each subject was given four preoperative tests, one each on Days 3, 6, 8, and 10, followed by surgery on Day 10 . Six postoperative tests were then given, one each on Days 13,15, 17, 20, 22, and 24. Food was removed from the cages of the deprived killers $24 \mathrm{~h}$ prior to each test, and was replaced when the dead mouse was removed from the cage after each trial.

Lesions were aimed at the trigeminal sensory root of each side using the following coordinates: $6.0 \mathrm{~mm}$ posterior to bregma, $3.1 \mathrm{~mm}$ lateral to the midline suture, and $8.5 \mathrm{~mm}$ below the dura. The incisor bar of the stereotaxis was positioned $5 \mathrm{~mm}$ above the interaural line. Cathodal lesions were made by passing $1.75 \mathrm{~mA}$ of current for $10 \mathrm{sec}$ with a rectal probe as the anode. In the control-operated rats (shams), the electrode was dropped to $1 \mathrm{~mm}$ above the lesion coordinates and no current was passed.

\section{Results}

All rats completed the kill within every 10 -min test session throughout the experiment. Lesions of the trigeminal sensory root did not affect initial attack speeds, but did decrease the speed and efficiency of killing (Figure 5). The natural killers differed from the food-deprived killers on all three behavioral measures. They were faster at killing, but slower at initially attacking and less effecient in their killing. Interpretation of the results of the statistical analyses was complicated by the unexpected outcome that four preoperative trials were not sufficient to stabilize the behavior of both the natural and fooddeprived killers. Thus, although the natural and food-deprived killers differed on all three behavioral measures following surgery, they differed significantly only on the speed to kill prior to surgery. The analyses of the preoperative behavioral data yielded three significant effects. The speeds of initial
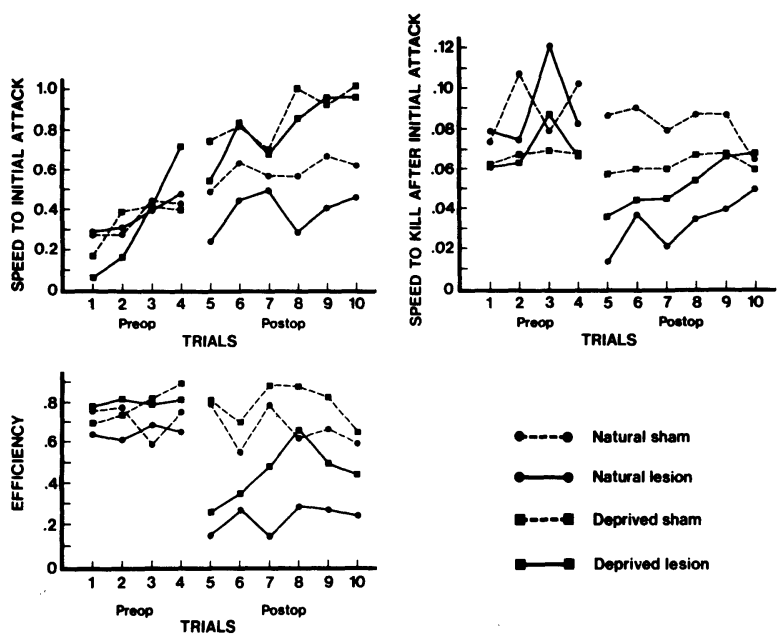

Figure 5. Mean speed (1/latency in seconds) of initial attack, speed to kill after the initial attack, and efficiency $(1 /$ number of attacks to complete the kill). Bilateral lesions were made in natural and food-deprived killers in the trigeminal sensory root at the level of the pons. Ten-minute trials were given once every 2-3 days, and there was a 3-day recovery period after surgery. attack increased over preoperative trials for both killer types $[\mathrm{F}(3,32)=10.10, \mathrm{p}<.001]$. The analysis of speeds to kill after the initial attack yielded a significant Lesion by Trials interaction $[F(3,32)=$ $3.33, p<.05]$. As suggested by the speed to kill curves in Figure 5 (upper right panel), this interaction is attributable to chance sample variability over the four preoperative trials. Ignoring this variability by averaging over the trials, the difference in killer type is significant $[F(1,34)=7.93, p<.01]$. The natural killers completed their kills faster than the food-deprived killers.

The analysis of the postoperative speeds to initially attack yielded only one significant effect, a killer type main effect $[F(1,34)=17.0, p<.001]$. The food-deprived killers attacked faster than the natural killers.

Analysis of the postoperative speeds to kill after the initial attack yielded a significant Killer Type by Lesion interaction $[\mathrm{F}(1,34)=12.1, \mathrm{p}<.01]$ and a significant Lesion by Trials interaction $[F(5,30)$ $=4.34, \mathrm{p}<.01]$. As suggested by the means in Figure 5, the trigeminal sensory root lesions decreased the speeds of killing of the natural killers more than the food-deprived killers. Recovery of the killing speed over postoperative trials would explain the Lesion by Trials interaction. Since this effect did not differentiate between natural and fooddeprived killers, this outcome also suggests that the food-deprived killers, as well, were affected by the lesions. A simple test of the speeds of killing on the first postoperative trial for the food-deprived killers with, compared to without, the trigeminal lesions supports this conclusion $[\mathrm{t}(16)=2.94$, $\mathrm{p}<.05]$.

Analysis of the efficiency ratios postoperatively yielded a significant Lesion by Trials interaction $[F(5,30)=4.07, p<.01]$. However, this interaction was primarily due to a relative improvement in efficiency of the lesion groups from the first to second postoperative trials. That is, the Lesion by Trials interaction for the difference in speeds from the first to second trials was significant $[F(1,34)$ $=10.19, \mathrm{p}<.01]$, while a multivariate test of the Lesion by Trials interaction for the postoperative trials ignoring the first trial was not significant $[\mathrm{F}(4,31)=2.58, \mathrm{p}>.05]$. There was a lesion main effect whether the first trial was included in the analysis $[F(1,34)=59.4, p<.001]$ or excluded from the analysis $[\mathrm{F}(1,34)=45.53, \mathrm{p}<.001]$. Trigeminal lesions decreased the efficiency of killing in both natural and food-deprived killers. The food-deprived killers were also found to be more efficient at killing than the natural killers $[F(1,34)=10.7$, $\mathrm{p}<.01$ ].

Reexamination of the postoperative data with covariate analyses, using the last preoperative score as 
the predictor variable, showed that the covariate was a significant predictor for all three behavioral measures. However, the results of the analysis were unchanged compared to the preceding analyses in all respects except one: the initial attack speeds were decreased by the trigeminal lesions according to a lesion main effect $[F(1,33)=5.48, p<.05]$. As inspection of Figure 5 suggests, the difference in speeds on the fourth preoperative trial for the two fooddeprived groups that subsequently received trigeminal or control surgery were in part responsible for the outcome. The results do not seem sufficient to warrant acceptance of an effect of the trigeminal lesions on speeds of initial attack.

In sum, trigeminal sensory root lesions did not clearly affect initiation of attack, but did decrease killing speed, and more in natural killers than in food-deprived killers. Killing efficiency was decreased by the lesions in natural and food-deprived killers. Recovery of the deficit in killing speeds was evident, but recovery of killing efficiency was only significant from the first to second postoperative trials.

General effects of the lesions. The rats with the trigeminal sensory root lesions were active and exhibited good muscle tone. They were able to gnaw dry food blocks and exhibited reflex chewing (with the exception of the one natural killer that was discarded: see General Methods). Body weights were not significantly different from the control values at the time that they were sacrificed for histoligical examination.

Damage to the trigemimal nerve was apparent at or anterior to its attachment to the pons (Figure 6). There was consistent bilateral destruction of some of the sensory portion of the nerve, but the precise location within this large sensory root was quite variable, as was the amount of destruction. There was no apparent relationship between the locus of sensory damage and magnitude of the killing deficiency. Partial unilateral damage of the motor root, which supplies the muscles of mastication via the mandibular division, was common. We could not find any relation between histological signs of motor damage and the degree of killing impairment. Slight
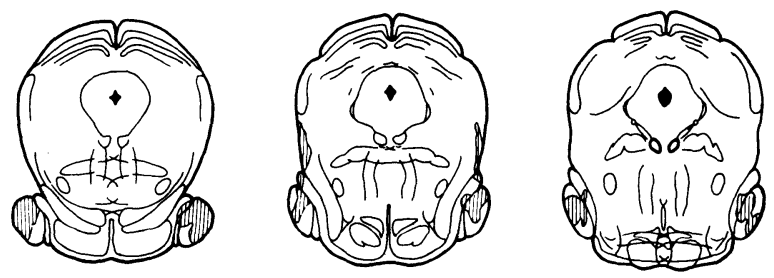

Figure 6. Schematic representation of tissue damage (shaded area) in a rat with a typical trigeminal root lesion. (Adapted from König and Klippel, 1963.) unilateral or bilateral damage to the middle cerebellar peduncle occurred frequently, but was not more common or extensive in the rats with the poorest performance. Electrode track damage to the lateral lemniscus occurred unilaterally in three rats.

\section{DISCUSSION}

Trigeminal lesions decreased the speed and efficiency of mouse killing whether the lesions were of the peripheral infraorbital branch, the sensory root, or the ventrobasal thalamic complex. In contrast, the initiation of the killing response, measured by the speed of the initial attack, was decreased only by VBm lesions. This deficit had largely recovered by the second postoperative week. Significant recovery of the deficit in killing speed occurred only in rats with sensory root lesions. Trigeminal damage thus appears to predominantly disrupt the somatosensory feedback involved in the guidance of the biting behavior.

The results of Experiment 2 differ from the finding of Gregoire and Smith (1975) that infraorbital transections reduce the frequency of attacking within 2-min test sessions by $50 \%$. Our infraorbital transections did not significantly reduce initial attack speeds, and in only two instances were 2 full minutes required for the completion of the kill. The differences in the subpopulations of rats used in the two studies appears to be at least partially responsible for the different results. While we used natural killers, Gregoire and Smith used rats that were made aggressive by starvation because they would not kill spontaneously. They then tested the rats following surgery under ad-lib conditions.

There is an apparent discrepancy between the Thor and Ghiselli (1975) finding of an increased latency of initial attack following lidocaine anesthesia of the mystacial vibrissae and our failure to find an effect of peripheral lesions on this measure. However, Thor and Ghiselli reported that the deficit emerged as a failure to deliver the killing bite after a normal pursuit and trapping of the mouse, and that those rats which failed to kill would do so on a subsequent day when retested under lidocaine treatment. Additionally, rats tested first with a placebo saline injection (delivered under partial ether anesthesia) were subsequently less affected by lidocaine treatment than those rats tested first with lidocaine treatment. These results suggest temporary effects of sensory denervaton that would have been alleviated in our chronic preparatons before the first postsurgical test occurred 3 days following surgery. Thor and Ghiselli concluded that the deficit produced by lidocaine anesthesia was in the execution of the motor attack rather than in motivation, as we have concluded from our results. 
Although studies have usually indicated that sensory deprivation other than perioral somatosensory denervation does not disrupt muricide (Alberts \& Friedman, 1972; Gregoire \& Smith, 1975; Karli et al., 1969), olfactory cues have been shown to play a role in inhibition of killing of rat pups by natural mouse-killers (Myer, 1964). Recently, Thorne and Thompson (1976) have shown that olfactory cues play a role in the differential killing of wild and domestic mice. The proportion of naive, male hooded rats killing mice was as low as $20 \%$ when the target mice were domesticated albinos, and was $100 \%$ when the target mice were recently captured wild mice. Deodorizing the mice tended to equalize the proportion of rats killing the different types of mice, with an increase in killing of the domesticated albinos being, apparently, the greater effect. They also found that removal of the vibrissae increased the median latency of killing without changing the proportions of rats killing wild or domestic mice. These results complement our own by emphasizing the relative unimportance of perioral cues in eventual elicitation of muricide without diminishing the importance of perioral cues in efficient guiding of muricide.

There appears to be a continuum of individual differences in the probability of muricidal behavior, such that natural vs. induced killing is an artificial dichotomy. Spontaneous killing is a function of a host of situational and experiential variables (e.g., Thorne \& Thompson, 1976). This is consistent with our proposition that the susceptibility of muricide to trigeminal damage is a function of the methods of selecting the experimental population and subsequently testing them. In the present study, a high proportion of spontaneous killers was found. Since it is certain that our rats had never experienced food deprivation prior to the experiment, a characteristic of the hooded rats or of the target we used must be responsible. With these natural killers, and with the use of three or four training trials prior to surgery, initial attack speeds were not affected except temporarily after VBm lesions.

The transient abolishment of attack behavior by lesions of the VBm may possible derive from the more complete somatosensory denervation of the face and mouth. Although we did not find such an effect with our sensory root lesions, we also did not find effects on eating and drinking comparable to those obtained with VBm lesions, and Miller (1977) has recently shown that extensive peripheral trigeminal deafferentation markedly decreases eating and drinking. The VBm lesion effect on elicitation of mouse-killing attack may therefore be due to either extensive trigeminal damage or to other damage produced by the lesions. The sensory root lesions were clearly not as extensive as intended, but did serve to demonstrate that comparable effects on the efficiency of mouse-killing behavior can be obtained in natural and food-deprived killers, with partial trigeminal deafferentation that does not affect food and water consumption.

The results of this study indicate that the infraorbital branch is not necessary for mouse-killing attack, and that various forms of peripheral and central trigeminal damage can cause relatively persistent disruption of the speed and efficiency of killing. This seems convincing of an essential trigeminal role in mouse-killing behavior in regard to sensorimotor coordination, but not motivation.

\section{REFERENCES}

Alberts, J. R., \& Friedman, M. I. Olfactory bulb removal but not anosmia increases emotionality and mouse-killing. Nature, 1972, 238, 454-455.

Greene, E. C. Anatomy of the rat. New York: Hafner, 1955.

Gregorre, S. E., \& SMITH, D. E. Mouse-killing in the rat: Effects of sensory deficits on attack behavior and stereotyped biting. Animal Behaviour, 1975, 23, 186-191.

Karli, P., Vergnes, M., \& Didiergeorges, F. Rat-mouse interspecific aggressive behavior and its manipulation by brain ablation and by brain stimulation. In S. Garattini \& E. B. Sigg (Eds.), Aggressive behaviour. New York: Wiley, 1969.

KönIG, J. F. R., \& KLIPPEL, R. A. The rat brain. Baltimore: Williams \& Wilkins, 1963.

Kulkarni, A. S. Satiation of instinctive mouse killing by rats. Psychological Record, 1968, 18, 385-388.

Marshall, J. F., Richardson, J. S., \& Teitelbaum, P. Nigrostriatal bundle damage and the lateral hypothalamic syndrome. Journal of Comparative and Physiological Psychology, 1974, 87, 808-830.

MiczeK, K. A., \& Grossman, S. P. Effects of septal lesions on inter- and intraspecies aggression in rats. Journal of Comparative and Physiological Psychology, 1972, 79, 37-45.

Miller, M. G. The effect of trigeminal deafferentation on food and water intake in the rat. Society for Neuroscience Abstracts, 1977, 3, 164. (Abstract)

MYER, J. S. Stimulus control of mouse-killing rats. Journal of Comparative and Physiological Psychology, 1964, 58, 112-117.

Myer, J. S., \& White, R. T. Aggressive motivation in the rat. Animal Behaviour, 1965, 13, 430-433.

Paul, L., Miley, W. M., \& Baenninger, R. Mouse-killing by rats: Roles of hunger and thirst in its initiation and maintenance. Journal of Comparative and Physiological Psychology, 1971, 76, 242-249.

Reyes, R., Finger, S., \& Frye, J. Serial thalamic lesions and tactile discrimination in the rat. Behavioral Biology, 1973, 8, 807-813.

SMITH, R. L. The ascending fiber projections from the principal sensory trigeminal nucleus in the rat. Journal of Comparative Neurology, 1973, 148, 423-446.

Thor, D. H., \& Ghiselli, W. B. Suppression of mouse killing and apomorphine-induced social aggression in rats by local anesthesia of the mystacial vibrissae. Journal of Comparative and Physiological Psychology, 1975, 88, 40-46.

Thorne, B. M., \& Thompson, M. E. The effect of different types of mice upon muricidal behavior in the Long-Evans rat. Physiological Psychology, 1976, 4, 238-246.

Van Hemel, P. E., \& Myer, J. S. Satiation of mouse-killing by rats in an operant situation. Psychonomic Science, 1970, 21, 129-130.

Zeigler, H. P., \& Karten, H. J. Central trigeminal structures and the lateral hypothalamic syndrome in the rat. Science, $1974,186,636-638$.

(Received for publication October 12, 1977: revision accepted April 3, 1978.) 